FUSARIUM TOXIN AS A SELECTION TOOL FOR TOLERANCE TO FUSARIUM CULMORUM IN WHEAT

\title{
C.H.A. SNIJDERS
}

Foundation for Agricultural Plant Breeding (SVP), Postbox 117, 6700 AC Wageningen (The Netherlands)

In a field trial 10 wheat genotypes with different resistance levels to ear blight were artificially inoculated with three pure isolates of Fusarium culmorum, collected in the Netherlands. The trial was carried out in two replicates. The ears were harvested and threshed by hand. The seeds were analyzed by TLC-analysis (ref. 1) for the presence of the mycotoxins deoxynivalenol (DON), 3-acetyl-deoxynivalenol, deacetoxyscipenol (DAS) and zearalenone. Only DON was demonstrated to be present in concentrations up to $48 \mathrm{ppm}$.

Although interactions between isolates and genotypes were observed, a path diagram as shown in Figure 1 indicates a relationship between DON and kernel weight reduction. All correlations were significant $(n=30, p=0.01)$.

To investigate the direct influence of DON on the growth of the different genotypes, seedlings were grown on a toxin containing medium as described in ref. 2. In ref. 2 a concentration of $5 \mathrm{ppm}$ DON was concluded to be optimal to screen genotypes for tolerance to DON. In the present trial 19 genotypes were grown at this concentration, in four replicates. The conditions for the trial were the same as described in ref. 2. For each genotype four controls without toxin were included.

The mean results of the four replicates are presented in Figure 2. For the controls only one response curve is shown, as no differences between the genotypes were found. The significant differences in response to DON between the 19 genotypes. Thus a causal effect of DON on plant growth was demonstrated. The reaction of the wheat seedling on $5 \mathrm{ppm}$ DON in vitro and the in vivo ear blight and kernel weight reduction were not significantly correlated, however.

From this first in vitro experiment we conclude that selection for 
DON-tolerance in vitro at this concentration does not automatically select for tolerance to ear blight. Selection at a higher concentration may open better prospects for selection which is being investigated.

\section{REFERENCES}

1 Snijders, C.H.A. \& J. Perkowski, Interaction between isolates of Fusarium culmorum and wheat genotypes for mycotoxin production. In preparation.

2 Snijders, C.H.A. The phytotoxic action of deoxynivalenol and zearalenone on wheat seedlings. These proceedings.

Fig. 1: Path diagram illustrating causal and possible relationships

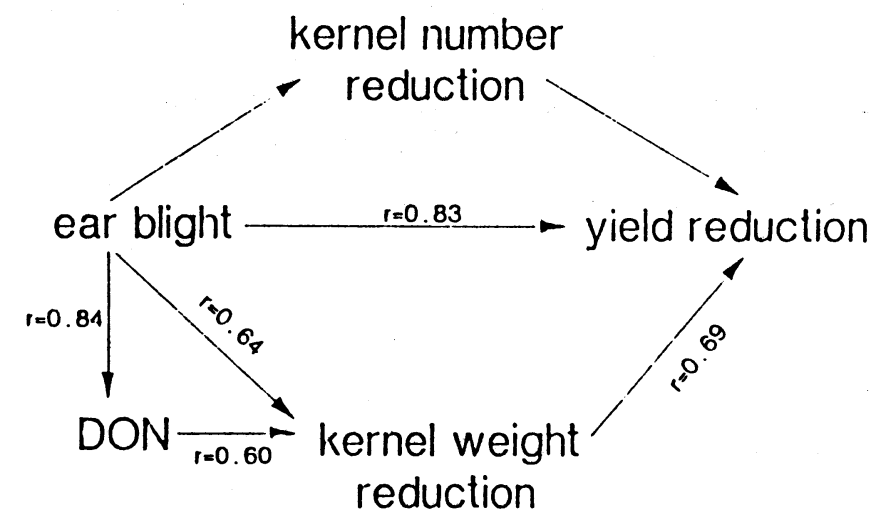

Fig. 2: Coleoptile growth of 19 wheat genotypes in vitro at a concentration of 5 ppm DON; 1 experiment, 4 replicates, $16{ }^{\circ} \mathrm{C}$ growing temperature

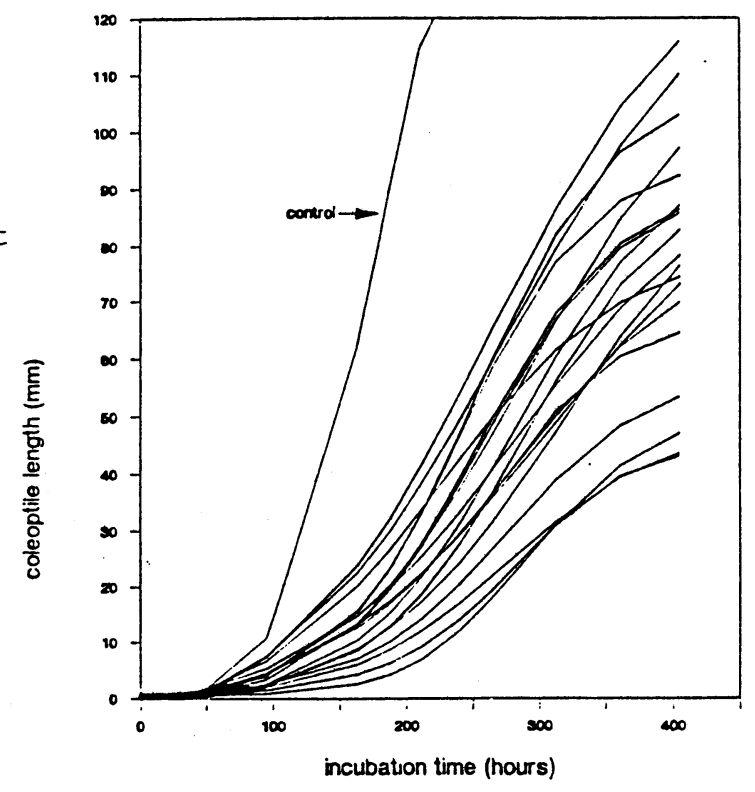

